\title{
Coherent Phonon Disruption and Lock-In During a Photoinduced Charge-Density-Wave Phase Transition
}

\author{
Spencer A. Reisbick ${ }^{\dagger}$, Yichao Zhang, Jialiang Chen, Paige E. Engen, and David J. Flannigan* \\ Department of Chemical Engineering and Materials Science, University of Minnesota, 421 \\ Washington Ave. SE, Minneapolis, MN 55455, USA
}

\begin{abstract}
Ultrafast manipulation of phases and phase domains in quantum materials is a key approach to unraveling and harnessing interwoven effects of charge and lattice degrees of freedom. In the intensely-studied charge-density-wave (CDW) material, $1 T-\mathrm{TaS}_{2}$, phonon coupling to periodic lattice distortions (PLDs) and atomically-incoherent picosecond structural phase transitions suggest transitional periods could exist for delayed onset of mode coherence. Here we find evidence for such a connection between displacively-excited coherent acoustic phonons and PLDs using 4D ultrafast electron microscopy. Following femtosecond optical excitation of an ultrathin crystal, a propagating hybridized mode is imaged emerging from linear defects within a $1-\mu \mathrm{m}$ region. Partial coherence and low amplitudes during onset of the incommensurate phase convert to higher-amplitude, increasingly-coherent oscillations as phase-growth stabilizes. The hybrid mode consists of large out-of-plane distortions coupled to basal-plane bond oscillations propagating at anomalously high velocities. The strongly-correlated behaviors observed here represent a potential means to control phase behaviors in quantum materials using defectengineered coherent-phonon seeding.
\end{abstract}

*Author to whom correspondence should be addressed.

Email: flan0076@umn.edu

Office: +1 612-625-3867 


\section{INTRODUCTION}

Key to understanding and controlling phase formation, domain size and motion, and transformation kinetics in strongly-correlated materials is development of comprehensive microscopic descriptions of charge, lattice, orbital, and spin dynamics spanning quantumdominated regimes and extending to mesoscopic spatiotemporal scales via coupling and conversion (1). This is especially the case for quantum materials displaying sensitive, weaklystable phases, intricate phase diagrams, and complex atomic-rearrangement trajectories that may or may not be coherent across critical points or during ultrafast excitation. The transition metal dichalcogenide (TMD) $\mathrm{TaS}_{2}$ is such a material, exhibiting a rich polymorphic, charge-density wave (CDW), and electronic phase space correlated to anomalies in bulk constitutive relations (2). In particular, the trigonal polymorph, with all $\mathrm{Ta}$ atoms in a distorted octahedral coordination environment (i.e., the $1 T$ phase), is the high-temperature stable form but is also metastable at room temperature when quenched (Fig. 1a) (3, 4).

Cooling the undistorted $1 T$ polymorph from high temperatures results in a series of firstorder CDW phase transitions $(5,6)$. Each phase is characterized by a signature periodic lattice distortion (PLD) driven by electron correlations and strong basal-plane electron-phonon coupling $(7,8)$. The transitions are correlated with dramatic changes in electronic structure owing to gaps opening on the Fermi surface, thus drawing broad, intense interest in the search for new quantum states and for significantly-advanced applications (9-14). For example, below $353 \mathrm{~K}$, an uncommon nearly-commensurate (NC) CDW phase forms and remains stable down to $\sim 180 \mathrm{~K}$. Unique to this phase is the grouping of CDWs into larger distorted hexagonal domains (HDs), with the basal-plane lattice, $\mathrm{CDW}$, and $\mathrm{HD}$ lengths and wave vectors $\left(\mathbf{k}_{L}, \mathbf{k}_{C D W}\right.$, and $\mathbf{k}_{H D}$, respectively) all being distinct (Fig. 1bc) $(6,15-19)$. Coherent scattering by the periodic modulations produces 
characteristic satellite reflections in electron diffraction, as well as hexagonal and striped contrast in satellite dark-field and bright-field transmission electron microscopy (TEM; Fig. 1 de) (20-25).
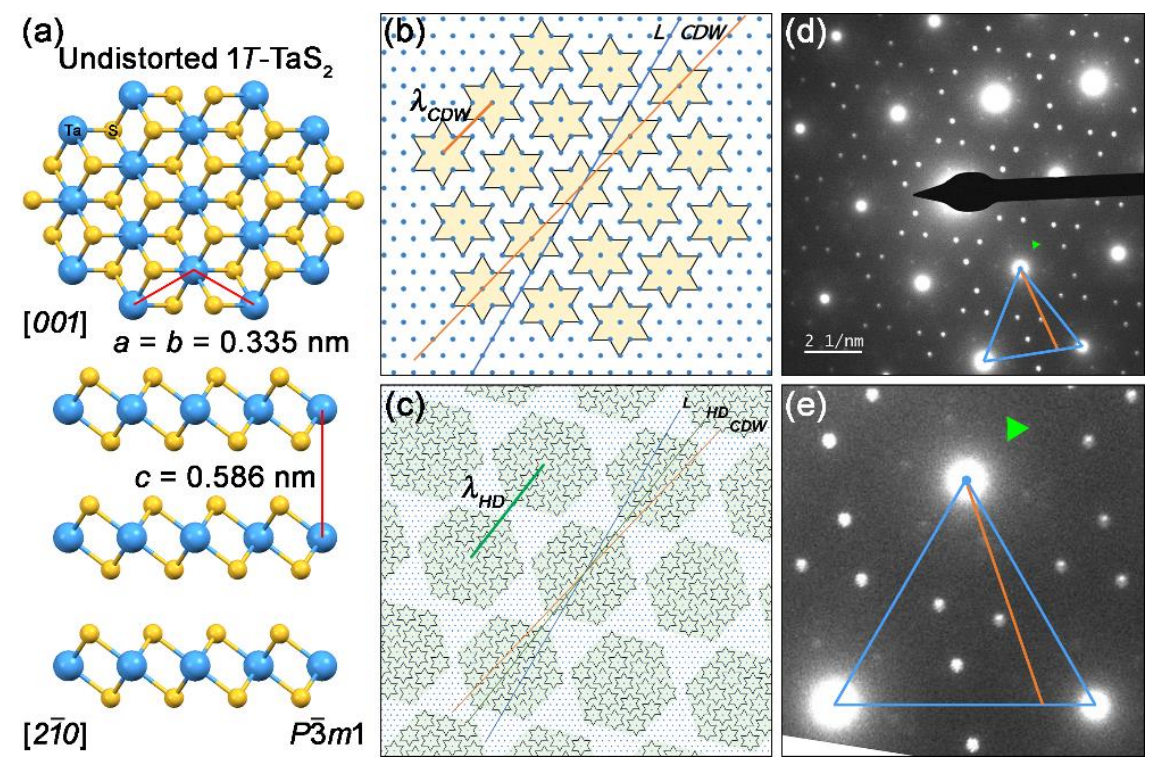

Figure 1. Structure of the uncommon NC phase of 1T-TaS2. (a) Undistorted structure viewed along the $[001]$ and $[2 \overline{1} 0]$ directions. Ta $=$ blue, $\mathrm{S}=$ yellow. Prepared using Mercury and the crystallographic information file for the $P \overline{3} m 1$ polymorph $(3,26)$. (b,c) Idealized real-space schematics of one layer of the NC phase viewed along [001] highlighting the CDW and HD distortions and domains ( $\mathrm{b}$ and $\mathrm{c}$, respectively). Only the Ta atoms are shown. One of three symmetry-related orientations of $\mathbf{k}_{C D W}, \mathbf{k}_{H D}$, and $\mathbf{k}_{L}$ are indicated. The characteristic CDW and HD wavelengths ( $\lambda_{C D W}$ and $\lambda_{H D}$, respectively) are also marked. Atomic-scale non-idealities are not shown for clarity (19). (d) Selected-area electron diffraction (SAED) of one of two degenerate orientations of the $\mathrm{NC}$ phase showing Bragg and strongly-modulated satellite peaks $(5,27)$. Weak coherent scattering from HDs is visible around nearest-neighbor satellite spots (green triangle). (e) Magnified and rotated view of the region shown in (d). Select Bragg (blue) and CDW (orange) reciprocal-space wave vectors are indicated. 
The CDW critical-point transitions are also correlated with anomalous changes in lattice parameters and the associated elastic tensor values (28-34). Under equilibrium conditions, this translates into abrupt modulation of constitutive relations describing acoustic and thermal energy transport. Little is known, however, about the corresponding responses during ultrafast photoexcitation. Under certain conditions, femtosecond excitation leads to complete suppression of the NC phase, nucleation of the incommensurate (IC) phase, and electron-lattice coupling in less than 5 ps $(35,36)$. Subsequent IC-phase dynamics consist of a fluence-dependent regime spanning tens to hundreds of picoseconds characterized by domain growth and coarsening (3739). An additional energy channel in ultrathin, freestanding crystals is the displacive excitation of coherent acoustic phonons (CAPs) also occurring over tens to hundreds of picoseconds (40, 41). Spatiotemporal commensurability with IC-phase domains and correlation lengths thus suggests propagating phonon modes should also display anomalous, strongly-correlated behaviors near critical points. Further, rapid suppression of the NC phase should pin CAP nucleation and wave vectors $\left(\mathbf{k}_{C A P}\right)$ to static structures, such as linear defects, owing to correlated initial symmetryraising and few-picosecond $c$-axis coupling to basal-plane modes (40-43).

Accordingly, we establish connections between linear defects, $\mathbf{k}_{C A P}$ and symmetries, and CDW PLD wave vectors during the photoinduced NC-to-incommensurate (IC) phase transition in $1 T-\mathrm{TaS}_{2}$ with 4D UEM imaging and diffraction (44-46). We find that in situ femtosecond photoexcitation of an ultrathin, freestanding crystal produces a hybridized CAP mode launched from a linear defect within a select $1-\mu \mathrm{m}$ ROI. Polarization coupling and oscillatory coherence are disrupted during the IC-phase growth period. The usual behavior of damped harmonic oscillation is not observed. Instead, wave amplitude increases as growth slows, suggesting domain 
correlation lengths approaching dimensionalities commensurate with the CAP wavelengths enhance the later propagating lattice distortions, particularly along the out-of-plane PLD direction. Correlating NC, IC, and lattice structural wave vectors to $\mathbf{k}_{C A P}$ confirms defects rather than groundstate PLDs are the dominant seeding and wave-guiding mechanism.

\section{MATERIALS AND METHODS}

Electron-transparent flakes were prepared via repeated exfoliation of a bulk $1 T-\mathrm{TaS}_{2}$ crystal (HQ Graphene) using adhesive tape (40-42). Flakes were transferred to an $\mathrm{NaCl}$ crystal (Ted Pella), followed by repeated washing with isopropyl alcohol and drying at $95{ }^{\circ} \mathrm{C}$. A 4-wt $\%$ solution of PMMA in anisole was then drop-cast onto the $\mathrm{NaCl}$ surface, followed by annealing at $95{ }^{\circ} \mathrm{C}$ for $10 \mathrm{~min}$. The resulting PMMA/specimen film was floated off in DI water and captured on a TEM Cu mesh grid. The specimen was then repeatedly washed with acetone to remove PMMA, salt, and residual adhesive. ROI thickness was measured with EELS to be $66 \mathrm{~nm}$ (47).

Ultrafast selected-area electron diffraction (U-SAED) and ultrafast bright-field imaging were conducted with a 200-kV UEM (FEI Tecnai Femto, Thermo Fisher Scientific) equipped with a 50- $\mu \mathrm{m}$ truncated $\mathrm{LaB}_{6}$ source (Applied Physics Technologies) set back $350 \mu \mathrm{m}$ from a custom 1-mm Wehnelt aperture $(48,49)$. The specimen was photoexcited (PHAROS, Light Conversion) in situ with fundamental 1.03- $\mu \mathrm{m}$ wavelength light, an average absorbed fluence of $4 \mathrm{~mJ} / \mathrm{cm}^{2}$, a $10-\mathrm{kHz}$ repetition rate, a pulse duration of $300 \mathrm{fs}$ (full-width at half-max, fwhm; GECO scanning autocorrelator, Light Conversion), and a spot-size of $100 \mu \mathrm{m}\left(e^{-2}\right.$ width; measured externally with a Newport 190-1100 nm Si CCD beam profiler and calculated extrapolation to the specimen position). This produces a nearly flat intensity profile across the $1-\mu \mathrm{m}$ diameter ROI. Photoelectrons were generated with fourth-harmonic light (4.8-eV photons). Harmonics of the 
fundamental were generated using a harmonics module (HIRO, Light Conversion), while fourthharmonic light was generated externally with standard nonlinear optics. Photoelectron packet duration was estimated to be $\sim 1$ ps based on electron-gun configuration, photon energy, laser spot size (i.e., effective emission area), laser fluence, and a 2.4-eV estimated $\mathrm{LaB}_{6}$ work function (49). Probed area was controlled with a post-specimen selected-area aperture centered on the optic axis. Time delays were established using a motorized linear translation stage (Aerotech PRO165LM with Soloist CP10-MXU controller). Stroboscopic pump-probe experiments were done with randomized 1-ps time steps via automated communication between the stage and camera (Gatan Orius) (50).

\section{RESULTS AND DISCUSSION}

Figure 2 displays the experimental concept and correlated UEM imaging and diffraction data obtained from the 1- $\mu \mathrm{m}$ ROI. Emphasis was placed on probing a select, micrometer-scale region in real and reciprocal space in order to correlate lattice and nanoscale dynamics to local symmetry-lowered morphologies (46). This is enabled by access to the back focal plane and image plane in the TEM base instrument via automated changes in lens excitations (Fig. 2a). Photoexcited CAPs manifest as propagating contrast wavefronts in an image series via local modulation of the scattering condition $(42,51-53)$. Correlating this with strong electron scattering by defects enables nanometer-femtosecond resolved imaging of nucleation points at atomic-scale discontinuities $(42,43)$. Such an occurrence is observed here in the $1-\mu \mathrm{m}$ ROI at nanometerpicosecond scales (Fig. 2bde). Unlike previous observations, however, both coherence and scattering strength increase relative to the initial response for wavefronts that emerge well after photoexcitation. This anomalous behavior also manifests in the lattice response (Fig. 2c-e), which 
can be determined by treating Bragg scattering-vector magnitudes and intensities $\left(\left|\boldsymbol{g}_{h k 0}\right|\right.$ and $I_{h k 0}$, respectively) kinematically (Fig. 2f; see the Supporting Information) (53). Here, both $\left|\boldsymbol{g}_{h k 0}\right|$ and $I_{h k 0}$ oscillate generally in-phase about average values, thus mapping out a repeating phase space indicative of coupled motion (Fig. 2g). As discussed below, however, phase agreement soon after photoexcitation is partially disrupted compared to later wavefronts, which also display anomalous vibration-amplitude increases. Note that Bragg spots were monitored in order to directly relate real-space and reciprocal-lattice CAP dynamics; with respect to constitutive relations at critical points, the much weaker CDW satellite spots display the same behaviors of interest.
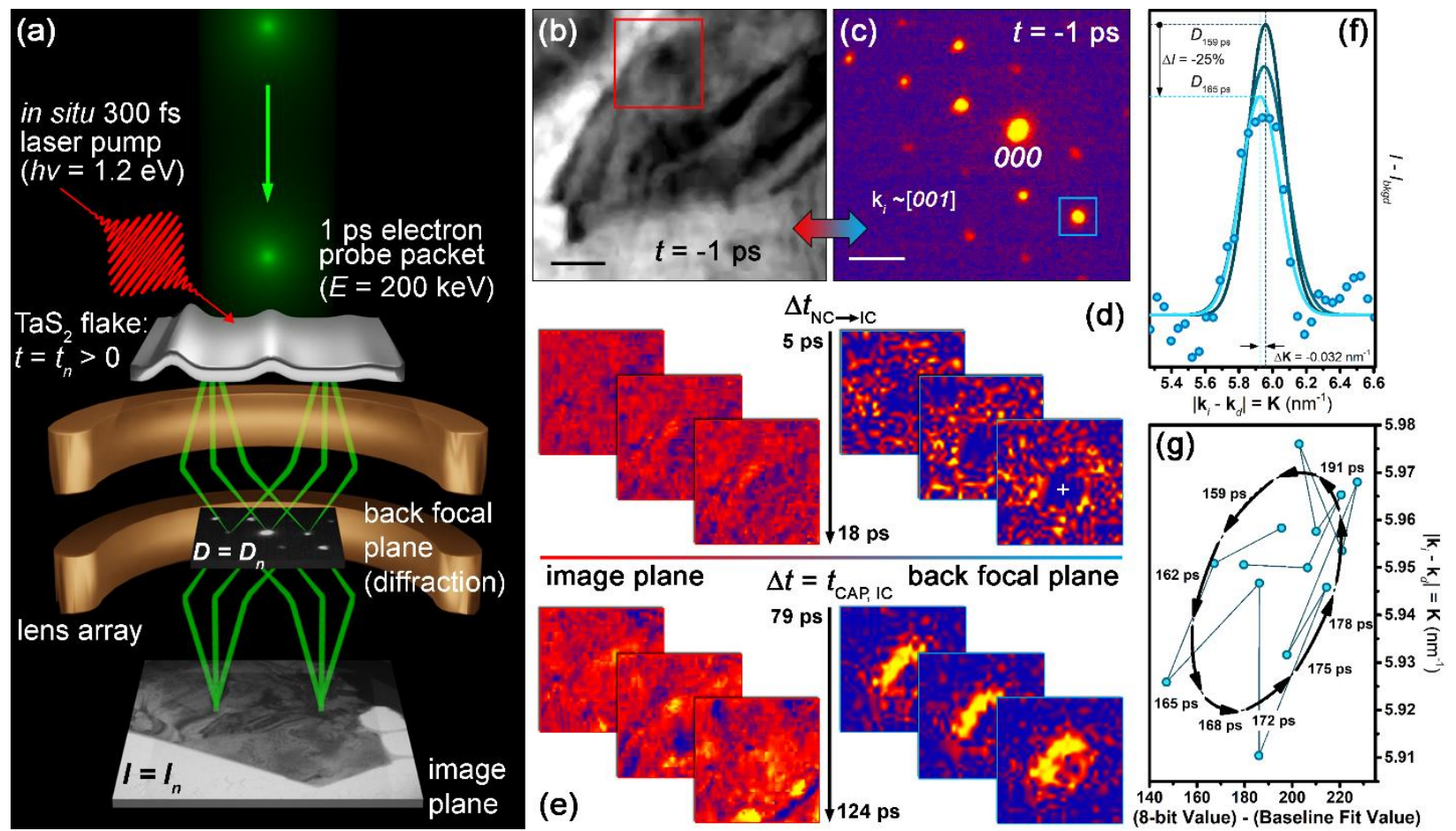

Figure 2. Spatiotemporal correlation of lattice and nanoscale CAPs with 4D UEM. (a) Concept of a single time-point acquisition, $t_{n}$. $D_{n}=$ back focal plane and $I_{n}=$ image plane at $t_{n}$. (b) UEM bright-field image ( $I_{-1}$ ps) of the $1-\mu \mathrm{m}$ ROI. Image has been smoothed and enhanced to better highlight strongly-scattering features. Scale bar $=250 \mathrm{~nm}$. (c) U-SAED pattern $\left(D_{-1 \mathrm{ps}}\right)$ 
from (b). Incident wave vector, $\mathbf{k}_{i}$, is nearly parallel to the [001] direction (see the Supporting Information). Arrow emphasizes complementary information. Scale bar $=3 \mathrm{~nm}^{-1}$. (d) Early $(\Delta t$ $=5-18$ ps) real- and reciprocal-space dynamics temporally correlated with the IC growth phase (36, 38). (e) Later dynamics ( $\Delta t=79-124 \mathrm{ps)}$ of the first coherent, large-amplitude CAP wavefront temporally correlated with slowing and stabilization of IC-phase growth. Image panels are normalized $\left(I_{n} / I_{-1}\right.$ ps $)$ to the red-boxed region in (b). Diffraction panels are differences of the blue-boxed scattering vector in (c) $\left(\left|D_{n}-D_{-1 \mathrm{ps}}\right|\right)$. (f) Select $\boldsymbol{g}_{h k 0}$ and $I_{h k 0}$ dynamics $\left(\mathbf{K}_{165 \mathrm{ps}}-\mathbf{K}_{159} \mathrm{ps}\right.$ $=-0.032 \mathrm{~nm}^{-1}, \mathbf{K}_{n}=$ magnitude difference between $\mathbf{k}_{i}$ and general scattering vector $\mathbf{k}_{d} ; I_{165 \mathrm{ps}}-I_{159}$ ps $=-25 \%$, baseline fit values $I_{b k g d}$ subtracted;). Data points shown for $D_{165}$ ps; peaks are Gaussian best fits. (g) Pixel-value (baseline subtracted) and $\Delta \mathbf{K}$ phase space for $159-200$ ps. Data points chronologically connected and temporal directional ellipse overlaid to guide the eye.

Determination of specimen photothermal response and correlation to characteristic NC-toIC timescales is needed before expanding on the results in Figure 2. For the pump laser settings, material properties (54-56), and crystal thickness and boundary conditions, simulation of singlepulse lattice temperature showed an increase from $293.15 \mathrm{~K}$ to just over $479 \mathrm{~K}$ within the first picosecond and cooling by less than one degree in the first $50 \mathrm{ps}$ (see the Supporting Information). This peak temperature is nearly identical to that calculated by Haupt et al. at $2 \mathrm{~mJ} / \mathrm{cm}^{2}$ (with different experimental parameters) (36). Here, a single-temperature model was used owing to the low $1 T-\mathrm{TaS}_{2}$ thermal conduction necessitating a fine mesh size and extremely tight tolerance (55). Latent heat of the NC-to-IC transition was also not incorporated owing to resulting instabilities (32). Thus, the simulated 1-ps rise here is not a reflection of the electron-phonon coupling time $(35,36)$. The single-pulse photothermal energy fully dissipates in less than $1 \mu \mathrm{s}$, well within the 
time between pulses $(100 \mu \mathrm{s})$. Note that the lower normalized photothermal temperature rise compared to the results of Haupt et al. can be attributed to different experimental and specimen parameters; a rough estimate based on total heat capacity and absorbed fluence indicates the temperature rise here should be $\sim 1.5$ times smaller, in agreement with observation.

Femtosecond photoexcitation of the NC-to-IC transition occurs via a multi-stage process (36-38). For conditions comparable to those here, electron-phonon coupling and complete suppression of the NC phase occurs within $\sim 1 \mathrm{ps,} \mathrm{whereas} \mathrm{the} \mathrm{IC} \mathrm{phase} \mathrm{nucleates} \mathrm{with} \mathrm{a} \mathrm{fluence-}$ independent time constant of $1.5 \mathrm{ps.} \mathrm{[Note} \mathrm{that} \mathrm{comparable} \mathrm{coupling} \mathrm{times} \mathrm{occur} \mathrm{at} \mathrm{the} \mathrm{lower}$ extreme of the NC phase range (35).] Nucleation is followed by fluence-dependent IC-phase growth or domain coarsening, with a time constant of 50-ps for the same quasi-equilibrium temperature reached here. The IC phase remains stable for several nanoseconds or longer before significant NC phase ground-state recovery begins (36). For moderate fluences resulting in mixed phases, a secondary IC-domain growth phase begins nanoseconds after initial stabilization (37). The timescale for initial IC-phase growth is dictated by repositioning of the basal-plane Ta atoms originally comprising the CDW PLDs, while secondary growth emerges from a disordered state and is ascribed to domain coarsening that follows a $\Delta t^{0.5}$ Lifshitz-Allen-Cahn growth law (37, 38). Increasing correlation lengths $[\xi(t)]$ determined from IC satellite-peak narrowing reflect the domain-size increase, though a growth mechanism (rather than coarsening) would not be expected to follow a $\Delta t^{0.5}$ power law (57).

Here, CAP symmetry, $\mathbf{k}_{C A P}$, and lattice distortions were determined from photoinduced modulation of $I_{h k 0}$ and $\boldsymbol{g}_{h k 0}$ using U-SAED (Fig. 3). The overall dynamics are dominated by an $f$ $=24-\mathrm{GHz}$ oscillation of both parameters. Analysis along azimuthal angles $(\gamma)$ of the scattering patterns reveals a single $\mathbf{k}_{C A P}$ oriented mainly along the [110] direction, with slight preference 
toward the $[\overline{1} 00]$ direction (Fig. 3ab). Smaller-amplitude $\boldsymbol{g}_{h k 0}$ oscillations at increasing $\gamma$ relative to $\mathbf{k}_{C A P}$ arise from vector-component contributions by the planar wavefronts. The dynamics reflect displacive excitation of a single, mixed-polarization hybridized mode having both longitudinal and transverse acoustic character (LA and TA, respectively) $(58,59)$. The response is not likely due to phonon mixing, as symmetry considerations indicate crossing of LA and TA branches should not occur along this direction (60). Interestingly, observation of CAP $d$-spacing oscillations (Fig. 3c) is uncommon despite PLDs having large-amplitude LA-mode character consisting of significant basal-plane atomic modulations $(8,40,61-65)$.
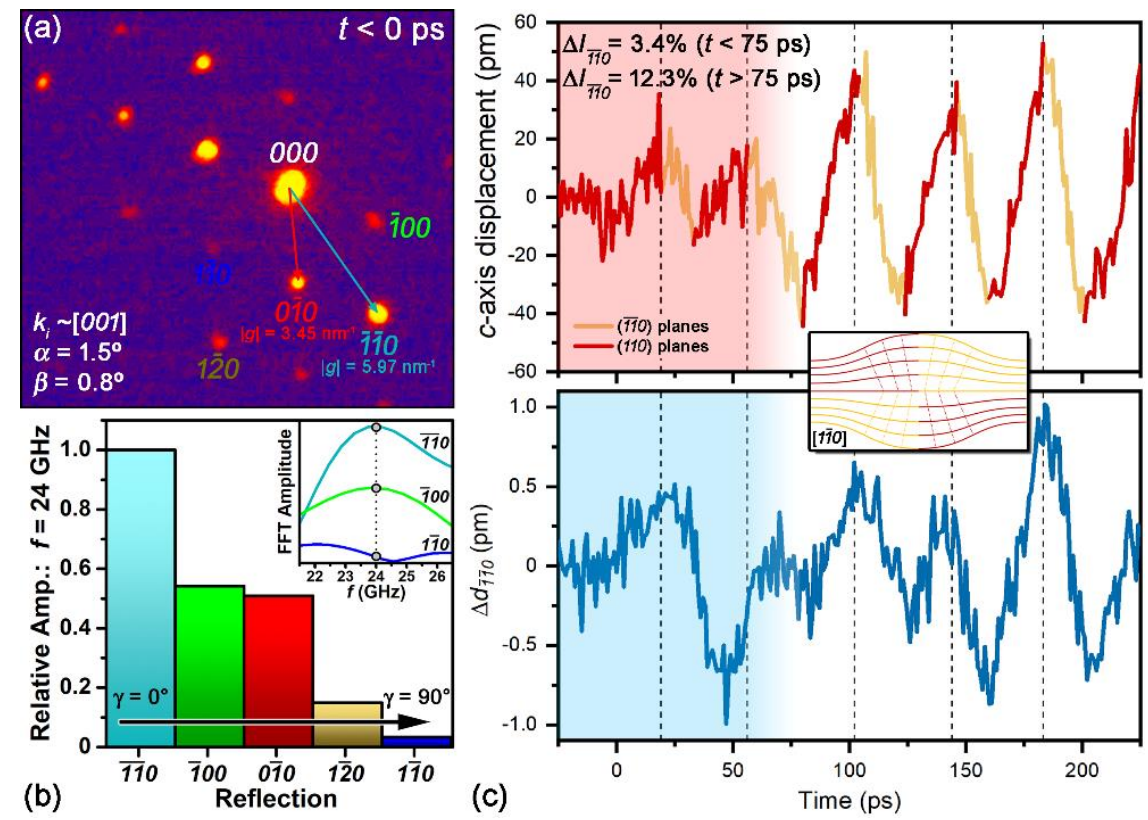

Figure 3. Ultrafast SAED of anomalous CAP dynamics during the NC-to-IC transition. (a) Representative pattern with select reflections and $|\boldsymbol{g}|$ indicated. Intensity and specimen orientation $\left(\alpha=1.5^{\circ}\right.$ and $\left.\beta=0.8^{\circ}\right)$ are such that IC satellite peaks are only faintly visible with contrast enhancement. (b) FFT amplitudes of $\boldsymbol{g}_{h k 0}$ oscillations normalized to $\boldsymbol{g}_{\overline{1} \overline{1} 0}$. Azimuthal angle $(\gamma)$ relative to $\boldsymbol{g}_{\overline{1} \overline{1} 0}$ is noted. Inset: FFTs of select $\boldsymbol{g}_{h k 0}$. (c) $c$-axis and $d_{\overline{1} \overline{1} 0^{-}}$-spacing dynamics. Dashed 
vertical lines mark approximate peak positions of the $c$-axis oscillation. Color gradients denote the low-amplitude, partially-coherent regime; $c$-axis oscillation amplitudes $\left(\Delta I_{\overline{1} \overline{1} 0}\right)$ before and after 75 ps are $3.4 \%$ and $12.3 \%$, respectively. Inset: illustration of the hybridized CAP symmetry viewed along the $[1 \overline{1} 0]$ direction.

As seen in Figure 3c, both Bragg-scattering parameters display anomalous behavior for $t<$ $\sim 75$ ps. The largest-amplitude and most-coherent lattice oscillations set-in following an initially low-amplitude, partially-coherent disrupted regime. This differs from CAP behaviors in nonCDW materials like $2 \mathrm{H}-\mathrm{MoS}_{2}$, where coherent $\mathrm{GHz}$ (early) and $\mathrm{MHz}$ (late) regimes are linked via a highly-incoherent transition period (66). The behavior and timescale suggest incoherent growth of the IC phase following photoexcitation disrupts fully-coherent formation and coupling of early CAP wavefronts (36-38). As phase growth slows and $\xi(t)$ increases over the first $\sim 100 \mathrm{ps,} \mathrm{the} \mathrm{IC}$ domains come into closer spatial commensurability with the CAP wavefront distortions. This behavior is in accordance with strong, first-order dependence of bulk constitutive properties on CDW phases, where discontinuities occur at critical points. Note that the anomalous behavior is not likely due to some form of CDW-amplitude enhancement (67). Instead, a phase locking of the polarized components and an increase in coupling strength occurs as basal-plane atomic order grows in the newly-formed IC phase.

The $\sim 50$-pm $c$-axis distortion is quite large, being a factor of $\sim 5$ greater than the largest Satom out-of-plane modulations in the NC phase (19). Along with the anomalous behavior, this suggests the transversely-polarized component is particularly susceptible to PLD modulation, indicating a potentially strongly-correlated relationship. A number of equilibrium and nonequilibrium behaviors also suggest the possibility of such an effect. For example, the $I \mathrm{~T}$

\section{Page 11 of 24}


polymorph exhibits particularly strong interplanar interactions via free-energy minimization through electrostatic and strain interactions of the CDW domains, as evidenced by comparable $C_{11}$ and $C_{33}$ elastic constants $(60,68)$. Similarly, Benedek et al. observed strong coupling of surface Rayleigh phonons to CDW PLDs in the commensurate (C) phase, as well as CDW-induced TA phonon propagation along the [110] basal-plane direction (69). This is thought to arise from sensitive modulation of the periodic electron density despite LA modes dominating electronphonon interactions at the Fermi surface (60). Correlated with the observed directionality is the photoinduced formation of anisotropic IC domains from the NC phase with different $\xi(t)$ along the [210] and [120] basal-plane directions (57). Further, the shear component of Rayleigh phonons in $2 \mathrm{H}-\mathrm{TaSe}_{2}$ is much smaller than the transverse component, and anomalous dispersion behaviors not due to a Kohn anomaly occur for the lowest-order TA mode ( $c$-axis polarization) propagating in the [hk0] direction in $1 T-\operatorname{TiSe}_{2}(70,71)$.

To connect the reciprocal-lattice dynamics to real-space nanoscale behaviors, UEM brightfield imaging was conducted on the same ROI (Fig. 4). Unlike in the U-SAED measurements, a delay of $\sim 100$ ps following photoexcitation occurs before a CAP wavetrain is resolved emerging from a static, extended but discrete crystal defect. Overlaying the diffraction pattern on the image confirms the single $\mathbf{k}_{C A P}$ deviates by a few degrees azimuthally from the [ $\left.\overline{1} \overline{1} 0\right]$ direction (Fig. $4 a$ ). Using vector-component analysis of a series of space-time contour plots $(51,52)$, the basal-plane velocity, $v_{C A P}$, was found to be an anomalously-high $4.6 \mathrm{~nm} / \mathrm{ps}$ (Fig. $4 \mathrm{bc}$ ). Increases in subsequent wavefront thickness in the contour plot (Fig. 4c) are due to an approximately linear movement of the crystal closer to the [001] zone axis - amplitudes of background-corrected scattering-vector intensities are mostly stable during this time ( $c f$. , Fig. 3c). This is an early signature of relaxation and branching to a whole-flake mechanical oscillation (66). In addition to $\mathbf{k}_{C A P}$, the $f=24-\mathrm{GHz}$

\section{Page 12 of 24}


wavetrain frequency also matches the Bragg-spot dynamics (Fig. 4d), revealing a CAP wavelength $\left(\lambda_{C A P}\right)$ of $190 \mathrm{~nm}\left(v_{C A P} / f\right)$. Altogether, this confirms the real-space dynamics are precisely those elucidated from the reciprocal-lattice response. Using an optical flow method spanning 20 ps (i.e., 0.5 CAP period), the extended defect was confirmed to be the CAP source (Fig. 4e). In-plane orientation of the defect relative to $\mathbf{k}_{C A P}$ suggests it also sets propagation direction; with little correlation to ground-state NC-phase PLDs (Fig. 4f). This shows that static defect structures dictate acoustic-phonon wave vectors during ultrafast manipulation of phase domains. (Note that we compare $\mathbf{k}_{C A P}$ to azimuthally-closest PLD wave vectors in the NC phase despite it being entirely suppressed within $\sim 1$ ps in order to determine if such features might seed and influence CAP propagation, particularly in the vicinity of static lattice discontinuities.)
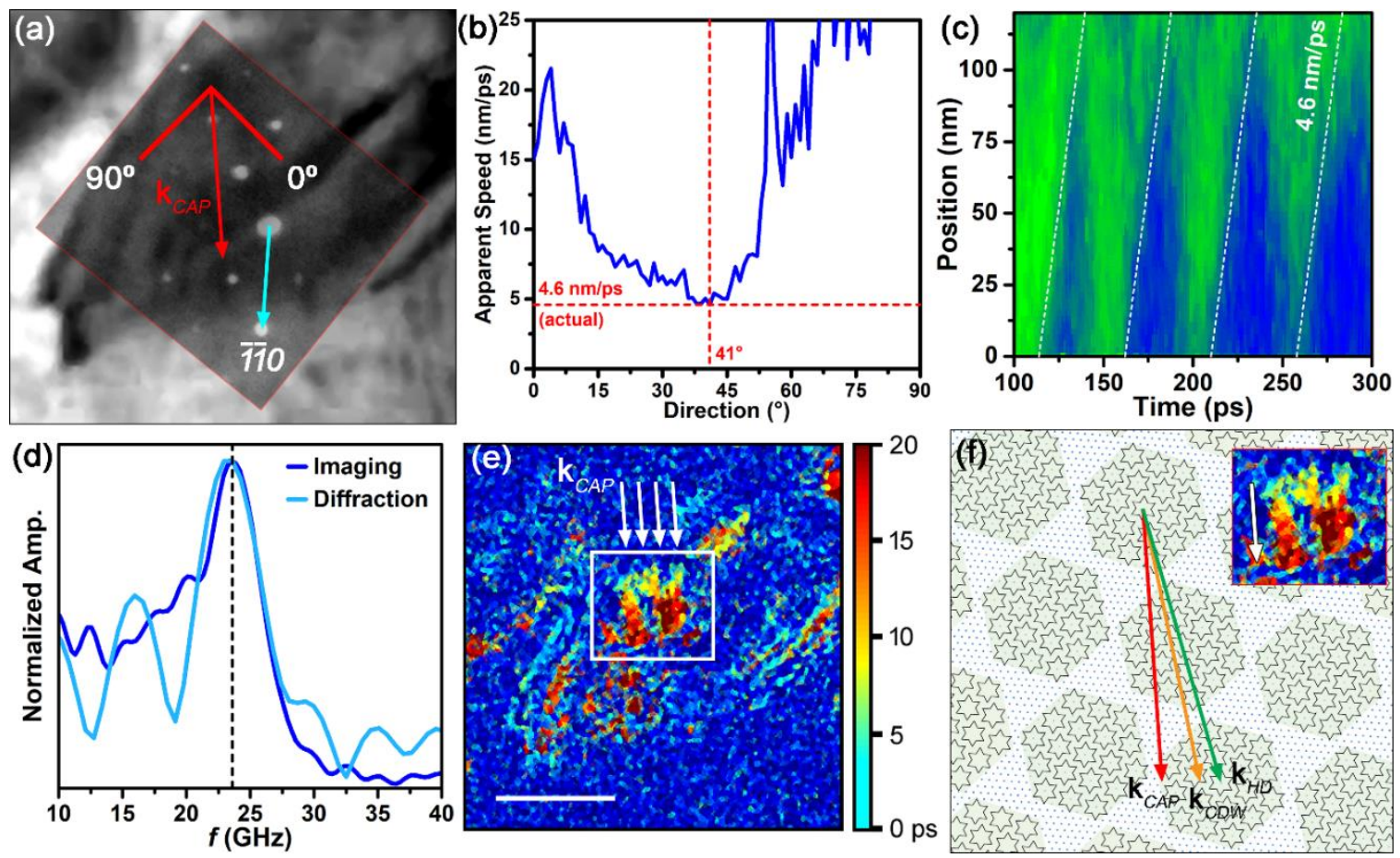

Figure 4. UEM imaging of defect-seeded CAP dynamics during the NC-to-IC transition. (a)

Orientation of real-space $\mathbf{k}_{C A P}$ relative to the crystal lattice (teal arrow is [ $\left.\overline{1} \bar{l} 0\right]$ direction) via an 
overlaid SAED pattern. (b) Apparent CAP speed versus direction within the ROI. Actual speed and direction were found to be $4.6 \mathrm{~nm} / \mathrm{ps}$ and $41^{\circ}$, respectively, within the defined coordinate region in (a). (c) Space-time contour plot of a 120-nm segment along $\mathbf{k}_{C A P}$. Each blue band is a single wavefront. (d) FFT of contrast oscillations compared to that of the $\overline{1} \overline{1} 0$ Bragg reflection. (e) Optical-flow analysis of the ROI for a 20-ps window containing CAP dynamics. Blue $=$ no motion, light blue $=0 \mathrm{ps}$ (nucleation), and increasingly hotter colors $=$ launch and propagation . White arrows denote $\mathbf{k}_{C A P}$ determined from vector analysis and U-SAED. Note the strong correlation with the temporal optical-flow direction, confirming the validity of the analyses. Scale bar $=250 \mathrm{~nm}$. (f) Comparison of $\mathbf{k}_{C A P}$ to $\mathbf{k}_{C D W}$ and $\mathbf{k}_{H D}$ in the pre-photoexcited NC phase. Inset: region in (e) with $\mathbf{k}_{C A P}$ (white arrow) adjacent to the temporal optical-flow gradient.

Though the defect structure could not be conclusively determined, the contrast and observed behaviors favor a step edge or crystal terrace. Indeed, coupling of $c$-axis and basal-plane CAPs has been shown to readily occur at step edges in TMDs (41-43). For example, in $2 \mathrm{H}-\mathrm{MoS}_{2}$, introduction of transverse character into propagating basal-plane modes occurs within 2.4 ps at few-layer step edges. Finite element simulations using indicate symmetry lowering at step edges leads to rapid, localized $c$-axis dephasing and picosecond-delayed vibrational coupling into the basal planes $(40,43)$. However, photoexcited $c$-axis distortion amplitudes at symmetry-lowered discontinuities are yet to be correlated to coupled basal-plane amplitudes. Thus, it is as yet unclear to what extent this might be influenced by ground-state PLDs to produce the large, $c$-axis polarized component observed here.

Incoherent IC-phase nucleation occurs prior to the increased CAP coherence and amplitude anomaly (compare Fig. 3c and Fig. 4c) (36-38). During this time, associated bright-field contrast 
oscillations are not clearly detected (or are not yet detectable). As initial phase growth slows and domain $\xi(t)$ begins to increase, PLD/CAP correlations will increase as well due to growing spatial commensurateness. For example, with ultrafast low-energy electron diffraction, it was found that the fluence- and time-dependent increase in the IC-phase $\xi$ reached $50 \mathrm{~nm}$ in 100 ps or slightly more than $25 \%$ of $\lambda_{C A P}(38)$. As a result, enhanced coupling to the IC domains would display a temporal response correlated to $\xi(t)$. The overall $\mathbf{k}_{C A P}$ behavior would then be determined by a combination of seed-defect orientation and IC domain growth (independent of initial NC PLD arrangements), depending upon how such static structures influence local phase kinetics. Comparisons to transient IC-phase anisotropic domain shape with respect to phonon wave guiding are intriguing (57). Indeed, picosecond acoustic-phonon trapping by nanoscale crystallites has been suggested to arise from dimensional commensurability and coupling, thus driving domainsymmetry enhancement via shear motion $(72,73)$.

The discussion above notwithstanding, absence of $\mathbf{k}_{C A P}$ wave guiding by basal-plane lattice and PLD wave vectors during the NC-to-IC transformation indicates the microscopic dynamics differ fundamentally from bulk Rayleigh surface modes. Further evidence of this is the anomalously-high $v_{C A P}$ (see Fig. 4c). For ultrathin crystals, the lowest-order Lamb mode having quasi-longitudinal character will have the highest velocity (29). However, previously-reported velocities of surface, plate, and bulk modes in $1 T-\mathrm{TaS}_{2}$ are all significantly lower than seen here $(29,58,60,74)$. For example, Ziebeck et al. measured bulk acoustic-phonon dispersion curves with neutron scattering at $300 \mathrm{~K}$ and calculated basal-plane LA velocities no higher than $3 \mathrm{~nm} / \mathrm{ps}$, while Jericho et al. reported a similar basal-plane plate-mode velocity of $3.1 \mathrm{~nm} / \mathrm{ps}$ using ultrasonic waves $(29,60)$. Harley and Fleury photoexcited Rayleigh waves in $1 T-\mathrm{TaS}_{2}$ at $300 \mathrm{~K}$ with $514.5-$ 
$\mathrm{nm}$ linearly-polarized continuous-wave laser light and measured surface velocities of $1.45 \mathrm{~nm} / \mathrm{ps}$ using Brillouin spectroscopy (58).

Importantly, bulk-mode basal-plane velocities in $1 T-\mathrm{TaS}_{2}$ could not be measured with Brillouin scattering owing to optical opacity, but measurements of other less-opaque TMDs (e.g., $2 H-\mathrm{TaSe}_{2}, 1 T-\mathrm{TiSe}_{2}$, and $\left.2 \mathrm{H}-\mathrm{NbSe}_{2}\right)$ returned values very similar to that measured here $(70,75)$. This may be a consequence of the large depth of field and in-focus, through-volume probing afforded by TEM, which in the case of UEM enables direct imaging of high-velocity bulk and plate volume modes not observable with optical spectroscopies in strongly-absorbing materials. For example, Nakamura et al. found good agreement between calculated and UEM-measured plate modes in $\mathrm{VTe}_{2}$, but they did observe an unexpected symmetry they ascribed to transient disruption of the CDW ordering (i.e., a photoinduced melting or "dissolution" of the CDW phase) (63). It is interesting to note that abrupt structural changes at the C-to-NC critical temperature result in acoustic emission, much like what occurs during diffusionless phase transitions and magnetoacoustic responses (76). Were similar emission to occur at the NC-to-IC transition, one wonders if the associated pulse might also interfere with CAP formation and launch.

Finally, though dedicated ultrafast diffraction experiments have been conducted on the NCto-IC transition $(35-39,77)$, inclusion of real-space measurements provides access to new insights (78). Here, this includes the specific nanoscale spatiotemporal characteristics of how the $1-\mu \mathrm{m}$ ROI switches between phases during photoexcitation, phonon excitation and diffuse heating, and subsequent cooling back through the critical point. For example, no obvious dispersion or anomalous behavior appears in the observed CAP wavefronts in Figure 4c despite abrupt changes in the pertinent constitutive properties occurring at the NC-IC critical point (29). One possibility is that the associated photoinduced transformation is simply not resolved, while another is that the 
response is ROI dependent. Indeed, dilute transient CDW pinning and de-pinning by defects or the presence of mixed-phase and mixed-state domain boundaries $(36,79)$ may vary specimen to specimen, thus producing specimen- and ROI-dependent atomic-potential modulation and illustrating the need for high spatiotemporal imaging resolutions (46).

In conclusion, we used correlative UEM bright-field imaging and U-SAED to establish relationships between propagating CAP dynamics, basal-plane structural wave vectors, and linear defects during the NC-to-IC phase transition in $1 T-\mathrm{TaS}_{2}$. Directionality of mostly in-phase intraand interlayer transient lattice-plane distortions was observed and quantified, and anomalous phase disruption and amplitude modulation was found to precisely coincide with the ultrafast photoinduced IC-phase growth period and increase in domain correlation length. Further, the photoexcited modes display hybridized polarization, are nucleated at linear defects, and propagate in the basal planes at anomalously high velocities. Altogether, these behaviors illustrate a potential means to control local, nanoscale phase domains and boundaries with photoinduced CAPs via symmetry-determined structural discontinuities and distortion-assisted depinning $(21,78,80)$.

\section{AUTHOR INFORMATION}

\section{Corresponding Author}

*Email: $\underline{\text { flan0076@umn.edu }}$

\section{ORCID}

David J. Flannigan: 0000-0002-1829-1868

Yichao Zhang: 0000-0003-1225-3278

†Present affiliation: Brookhaven National Laboratory, Upton, NY

\section{Author Contributions}


SR contributions were formal analysis, investigation, methodology, software, validation, visualization, writing - original draft, and writing - review and editing. YZ contributions were formal analysis, methodology, visualization, writing - original draft, and writing - review and editing. JC contributions were formal analysis, methodology, and visualization. PE contribution was investigation. DF contributions were conceptualization, funding acquisition, methodology, project administration, resources, supervision, visualization, writing - original draft, and writing - review and editing. See the CASRAI CRediT taxonomy for definitions of contributor roles.

\section{Funding}

This material is based upon work supported by the National Science Foundation under Grant No. DMR-1654318. This work was partially supported by the National Science Foundation through the University of Minnesota MRSEC under Award Number DMR-2011401. Acknowledgment is made to the Donors of the American Chemical Society Petroleum Research Fund for partial support of this research. Part of this work was carried out in the College of Science and Engineering Characterization Facility, University of Minnesota, which has received capital equipment funding from the NSF through the UMN MRSEC program under Award Number DMR-2011401. JC was supported in part by the U.S. Department of Energy through the UMN Center for Quantum Materials under Grant No. DE-SC-0016371. YZ acknowledges support from the Louise T. Dosdall Fellowship.

\section{Notes}

The authors declare no competing financial interest.

\section{REFERENCES}

1. F. Giustino, J. H. Lee, F. Trier, M. Bibes, S. M. Winter, R. Valentí, Y.-W. Son, L. Taillefer, C. Heil, A. I. Figueroa, B. Plaçais, Q. Wu, O. V. Yazyev, E. P. A. M. Bakkers, J. Nygård, P. Forn-Díaz, S. De Franceschi, J. W. McIver, L. E. F. F. Torres, T. Low, A. Kumar, R. Galceran, S. O. Valenzuela, M. V. Costache, A. Manchon, E.-A. Kim, G. R. Schleder, A. 
Fazzio, S. Roche, The 2021 Quantum Materials Roadmap. J. Phys.: Mater. 3, 042006 (2021).

2. J. A. Wilson, F. J. Di Salvo, S. Mahajan, Charge-Density Waves and Superlattices in the Metallic Layered Transition Metal Dichalcogenides. Adv. Phys. 24, 117-201 (1975).

3. G. Hägg, N. Schönberg, X-ray Studies of Sulfides of Titanium, Zirconium, Niobium, and Tantalum. Ark. Kemi 7, 371-380 (1954).

4. $\quad$ F. Jellinek, The System Tantalum-Sulfur. J. Less-Common Met. 4, 9-15 (1962).

5. C. B. Scruby, P. M. Williams, G. S. Parry, The Role of Charge Density Waves in Structural Transformations of $1 \mathrm{~T} \mathrm{TaS} 2$. Philos. Mag. 31, 255-274 (1975).

6. R. E. Thomson, B. Burk, A. Zettl, J. Clarke, Scanning Tunneling Microscopy of the Charge-Density-Wave Structure in $1 T$-TaS 2 . Phys. Rev. B 49, 16899-16916 (1994).

7. H. W. Myron, A. J. Freeman, Electronic Structure and Fermi-Surface-Related Instabilities in $1 T-\mathrm{TaS}_{2}$ and $1 T-\mathrm{TaSe}_{2}$. Phys. Rev. B 11, 2735-2739 (1975).

8. J. C. Petersen, S. Kaiser, N. Dean, A. Simoncig, H. Y. Liu, A. L. Cavalieri, C. Cacho, I. C. E. Turcu, E. Springate, F. Frassetto, L. Poletto, S. S. Dhesi, H. Berger, A. Cavalleri, Clocking the Melting Transition of Charge and Lattice Order in $1 T-\mathrm{TaS}_{2}$ with Ultrafast Extreme-Ultraviolet Angle-Resolved Photoemission Spectroscopy. Phys. Rev. Lett. 107, $177402(2011)$.

9. A. H. Thompson, F. R. Gamble, J. F. Revelli, Transitions Between Semiconducting and Metallic Phases in $1 T \mathrm{TaS}_{2}$. Solid State Commun. 9, 981-985 (1971).

10. L. F. Mattheiss, Band Structures of Transition-Metal-Dichalcogenide Layer Compounds. Phys. Rev. B 8, 3719-3740 (1973).

11. L. Stojchevska, I. Vaskivskyi, T. Mertelj, P. Kusar, D. Svetin, S. Brazovskii, D. Mihailovic, Ultrafast Switching to a Stable Hidden Quantum State in an Electronic Crystal. Science 344, 177-180 (2014).

12. M. Yoshida, R. Suzuki, Y. Zhang, M. Nakano, Y. Iwasa, Memristive Phase Switching in Two-Dimensional 1T-TaS 2 Crystals. Sci. Adv. 1, e1500606 (2015).

13. I. Vaskivskyi, I. A. Mihailovic, S. Brazovskii, J. Gospodaric, T. Mertelj, D. Svetin, P. Sutar, D. Mihailovic, Fast Electronic Resistance Switching Involving Hidden Charge Density Wave States. Nat. Commun. 7, 11442 (2016).

14. A. K. Geremew, S. Rumyantsev, F. Kargar, B. Debnath, A. Nosek, M. A. Bloodgood, M. Bockrath, T. T. Salguero, R. K. Lake, A. A. Balandin, Bias-Voltage Driven Switching of the Charge-Density-Wave and Normal Metallic Phases in $1 \mathrm{~T}-\mathrm{TaS}_{2}$ Thin-Film Devices. ACS Nano 13, 7231-7240 (2019). 
15. K. Nakanishi, H. Shiba, Domain-Like Incommensurate Charge-Density-Wave States and the First-Order Incommensurate-Commensurate Transitions in Layered Tantalum Dichalcogenides. I. 1T-Polytype. J. Phys. Soc. Jpn. 43, 1839-1847 (1977).

16. A. Yamamoto, Hexagonal Domainlike Structure in $1 T-\mathrm{TaS}_{2}$. Phys. Rev. B 27, 7823-7826 (1983).

17. X. L. Wu, C. M. Lieber, Hexagonal Domain-Like Charge Density Wave Phase of $\mathrm{TaS}_{2}$ Determined by Scanning Tunneling Microscopy. Science 243, 1703-1705 (1989).

18. J. A. Wilson, Solution to the $1 \mathrm{~T}_{2}$ Discommensurate State of 1T-TaS 2 . An Example of Rotated Hexagonal Discommensuration. J. Phys.-Condes. Matter 2, 1683-1704 (1990).

19. A. Spijkerman, J. L. L. de Boer, A. Meetsma, G. A. Wiegers, S. Van Smaalen, X-ray Crystal-Structure Refinement of the Nearly Commensurate Phase of $1 T-\mathrm{TaS}_{2}$ in $(3+2)$ Dimensional Superspace. Phys. Rev. B 56, 13757-13767 (1997).

20. J. A. Wilson, A. Yoffe, The Transition Metal Dichalcogenides Discussion and Interpretation of the Observed Optical, Electrical and Structural Properties. Adv. Phys. 18, 193-335 (1969).

21. J. van Landuyt, G. van Tendeloo, S. Amelinckx, Electron Diffraction Study of Inter- and Intrapolytypic Phase Transitions in Transition Metal Dichalcogenides. III. Complementary Diffraction Studies and Lattice Imaging of the Deformation Waves. Phys. Status Solidi A 36, 757-777 (1976).

22. C. H. Chen, J. M. Gibson, R. M. Fleming, Direct Observation of Charge-Density-Wave Discommensurations and Dislocations in 2H-TaSe 2. Phys. Rev. Lett. 47, 723-725 (1981).

23. K. K. Fung, S. McKernan, J. W. Steeds, J. A. Wilson, Broken Hexagonal Symmetry in the Locked-in State of $2 \mathrm{Ha}_{-} \mathrm{TaSe}_{2}$ and the Discommensurate Microstructure of its Incommensurate CDW States. J. Phys. C: Solid State Phys. 14, 5417-5432 (1981).

24. C. H. Chen, J. M. Gibson, R. M. Fleming, Microstructure in the Incommensurate and the Commensurate Charge-Density-Wave States of $2 \mathrm{H}-\mathrm{TaSe}_{2}$ : A Direct Observation by Electron Microscopy. Phys. Rev. B 26, 184-205 (1982).

25. M. Kuwabara, M. Tomita, H. Hashimoto, H. Endoh, Direct Observation of the Superstructure of the Nearly Commensurate Phase in $1 \mathrm{~T}-\mathrm{TaS}_{2}$ by High-Resolution Electron Microscopy. Phys. Status Solidi A 96, 39-51 (1986).

26. C. F. Macrae, I. Sovago, S. J. Cottrell, P. T. A. Galek, P. McCabe, E. Pidcock, M. Platings, G. P. Shields, J. S. Stevens, M. Towler, P. A. Wood, Mercury 4.0: From Visualization to Analysis, Design and Prediction. J. Appl. Crystallogr. 53, 226-235 (2020).

27. J. van Landuyt, G. van Tendeloo, S. Amelinckx, Electron Diffraction Study of Inter- and Intrapolytypic Phase Transitions in Transition Metal Dichalcogenides. I. Electron Diffraction Patterns. Phys. Status Solidi A 26, 359-376 (1974). 
28. F. L. Givens, G. E. Fredericks, Thermal Expansion of $\mathrm{NbSe}_{2}$ and $\mathrm{TaS}_{2}$. J. Phys. Chem. Solids 38, 1363-1365 (1977).

29. M. H. Jericho, A. M. Simpson, R. F. Frindt, Velocity of Ultrasonic Waves in $2 \mathrm{H}-\mathrm{NbSe}_{2}$, 2H-TaS 2 , and $1 T-\mathrm{TaS}_{2}$. Phys. Rev. B 22, 4907-4914 (1980).

30. O. Sezerman, A. M. Simpson, M. H. Jericho, Thermal Expansion of IT-TaS 2 and 2H$\mathrm{NbSe}_{2}$. Solid State Commun. 36, 737-740 (1980).

31. A. Suzuki, R. Yamamoto, M. Doyama, H. Mizubayashi, S. Okuda, K. Endo, S. Gonda, Elastic Anomalies in 1T-TaS 2 . Solid State Commun. 49, 1173-1176 (1984).

32. S. C. Bayliss, A. M. Ghorayeb, D. R. P. Guy, Thermal and Transport Evidence for a Phase Transition in 1T-TaS 2 Observed at $282 \mathrm{~K}$ Upon Warming. J. Phys. C: Solid State Phys. 17, L533-L537 (1984).

33. E. Sato, K. Ohtake, R. Yamamoto, M. Doyama, K. Endo, Positron Annihilation Studies on CDW Transitions in Tantalum Dichalcogenides. Solid State Commun. 54, 45-48 (1985).

34. A. Suzuki, M. Koizumi, M. Doyama, Thermal Evidences for Successive CDW Phase Transitions in 1T-TaS 2 . Solid State Commun. 53, 201-203 (1985).

35. M. Eichberger, H. Schafer, M. Krumova, M. Beyer, J. Demsar, H. Berger, G. Moriena, G. Sciaini, R. J. Miller, Snapshots of Cooperative Atomic Motions in the Optical Suppression of Charge Density Waves. Nature 468, 799-802 (2010).

36. K. Haupt, M. Eichberger, N. Erasmus, A. Rohwer, J. Demsar, K. Rossnagel, H. Schwoerer, Ultrafast Metamorphosis of a Complex Charge-Density Wave. Phys. Rev. Lett. 116, 016402 (2016).

37. C. Laulhé, T. Huber, G. Lantz, A. Ferrer, S. O. Mariager, S. Grübel, J. Rittmann, J. A. Johnson, V. Esposito, A. Lübcke, L. Huber, M. Kubli, M. Savoini, V. L. R. Jacques, L. Cario, B. Corraze, E. Janod, G. Ingold, P. Beaud, S. L. Johnson, S. Ravy, Ultrafast Formation of a Charge Density Wave state in $1 T-\mathrm{TaS}_{2}$ : Observation at Nanometer Scales Using Time-Resolved X-Ray Diffraction. Phys. Rev. Lett. 118, 247401 (2017).

38. S. Vogelgesang, G. Storeck, J. G. Horstmann, T. Diekmann, M. Sivis, S. Schramm, K. Rossnagel, S. Schäfer, C. Ropers, Phase Ordering of Charge Density Waves Traced By Ultrafast Low-Energy Electron Diffraction. Nat. Phys. 14, 184-190 (2018).

39. G. Storeck, J. G. Horstmann, T. Diekmann, S. Vogelgesang, G. von Witte, S. V. Yalunin, K. Rossnagel, C. Ropers, Structural Dynamics of Incommensurate Charge-Density Waves Tracked By Ultrafast Low-Energy Electron Diffraction. Struct. Dyn. 7, 034304 (2020).

40. D. R. Cremons, D. A. Plemmons, D. J. Flannigan, Defect-Mediated Phonon Dynamics in $\mathrm{TaS}_{2}$ and $\mathrm{WSe}_{2}$. Struct. Dyn. 4, 044019 (2017). 
41. S. A. Reisbick, Y. C. Zhang, D. J. Flannigan, Influence of Discrete Defects on Observed Acoustic-Phonon Dynamics in Layered Materials Probed with Ultrafast Electron Microscopy. J. Phys. Chem. A 124, 1877-1884 (2020).

42. D. R. Cremons, D. A. Plemmons, D. J. Flannigan, Femtosecond Electron Imaging of Defect-Modulated Phonon Dynamics. Nat. Commun. 7, 11230 (2016).

43. Y. Zhang, D. J. Flannigan, Observation of Anisotropic Strain-Wave Dynamics and FewLayer Dephasing in $\mathrm{MoS}_{2}$ with Ultrafast Electron Microscopy. Nano Lett. 19, 8216-8224 (2019).

44. A. H. Zewail, Four-Dimensional Electron Microscopy. Science 328, 187-193 (2010).

45. D. J. Flannigan, A. H. Zewail, 4D Electron Microscopy: Principles and Applications. Acc. Chem. Res. 45, 1828-1839 (2012).

46. D. A. Plemmons, P. K. Suri, D. J. Flannigan, Probing Structural and Electronic Dynamics with Ultrafast Electron Microscopy. Chem. Mater. 27, 3178-3192 (2015).

47. R. F. Egerton, S. C. Cheng, Measurement of Local Thickness by Electron Energy-Loss Spectroscopy. Ultramicroscopy 21, 231-244 (1987).

48. E. Kieft, K. B. Schliep, P. K. Suri, D. J. Flannigan, Communication: Effects of ThermionicGun Parameters on Operating Modes in Ultrafast Electron Microscopy. Struct. Dyn. 2, $051101(2015)$.

49. D. A. Plemmons, D. J. Flannigan, Ultrafast Electron Microscopy: Instrument Response from the Single-Electron to High Bunch-Charge Regimes. Chem. Phys. Lett. 683, 186-192 (2017).

50. D. X. Du, S. A. Reisbick, D. J. Flannigan, UEMtomaton: A Source-Available Platform to Aid in Start-up of Ultrafast Electron Microscopy Labs. Ultramicroscopy 223, 113235 (2021).

51. D. R. Cremons, D. X. Du, D. J. Flannigan, Picosecond Phase-Velocity Dispersion of Hypersonic Phonons Imaged with Ultrafast Electron Microscopy. Phys. Rev. Mater. 1, 073801 (2017).

52. E. J. VandenBussche, D. J. Flannigan, High-Resolution Analogue of Time-Domain Phonon Spectroscopy in the Transmission Electron Microscope. Philos. Trans. R. Soc. A 378, 20190598 (2020).

53. D. X. Du, D. J. Flannigan, Imaging Phonon Dynamics with Ultrafast Electron Microscopy: Kinematical and Dynamical Simulations. Struct. Dyn. 7, 024103 (2020).

54. A. R. Beal, H. P. Hughes, W. Y. Liang, The Reflectivity Spectra of Some Group VA Transition Metal Dichalcogenides. J. Phys. C: Solid State Phys. 8, 4236-4248 (1975). 
55. M. D. Núñez-Regueiro, J. M. Lopez-Castillo, C. Ayache, Thermal Conductivity of $1 T$ $\mathrm{TaS}_{2}$ and 2H-TaSe2. Phys. Rev. Lett. 55, 1931-1934 (1985).

56. M. Kratochvilova, A. D. Hillier, A. R. Wildes, L. Wang, S.-W. Cheong, J.-G. Park, The Low-Temperature Highly Correlated Quantum Phase in the Charge-Density-Wave 1T$\mathrm{TaS}_{2}$ Compound. npj Quantum Mater. 2, 42 (2017).

57. G. Lantz, C. Laulhe, S. Ravy, M. Kubli, M. Savoini, K. Tasca, E. Abreu, V. Esposito, M. Porer, A. Ciavardini, L. Cario, J. Rittmann, P. Beaud, S. L. Johnson, Domain-Size Effects on the Dynamics of a Charge Density Wave in 1T-TaS 2. Phys. Rev. B 96, 224101 (2017).

58. R. T. Harley, P. A. Fleury, Surface Brillouin Scattering from Layered Metals and Semimetals. J. Phys. C: Solid State Phys. 12, L863-L868 (1979).

59. H. J. Zeiger, J. Vidal, T. K. Cheng, E. P. Ippen, G. Dresselhaus, M. S. Dresselhaus, Theory for Displacive Excitation of Coherent Phonons. Phys. Rev. B 45, 768-778 (1992).

60. K. R. A. Ziebeck, B. Dorner, W. G. Stirling, R. Schollhorn, Kohn Anomaly in the $1 \mathrm{~T}_{2}$ Phase of $\mathrm{TaS}_{2}$. J. Phys. F: Met. Phys. 7, 1139-1143 (1977).

61. L. L. Wei, S. S. Sun, C. Guo, Z. W. Li, K. Sun, Y. Liu, W. J. Lu, Y. P. Sun, H. F. Tian, H. X. Yang, J. Q. Li, Dynamic Diffraction Effects and Coherent Breathing Oscillations in Ultrafast Electron Diffraction in Layered 1T-TaSeTe. Struct. Dyn. 4, 044012 (2017).

62. A. Nakamura, T. Shimojima, M. Matsuura, Y. Chiashi, M. Kamitani, H. Sakai, S. Ishiwata, H. Li, A. Oshiyama, K. Ishizaka, Evaluation of Photo-Induced Shear Strain in Monoclinic $\mathrm{VTe}_{2}$ by Ultrafast Electron Diffraction. Appl. Phys. Express 11, 092601 (2018).

63. A. Nakamura, T. Shimojima, Y. Chiashi, M. Kamitani, H. Sakai, S. Ishiwata, H. Li, K. Ishizaka, Nanoscale Imaging of Unusual Photoacoustic Waves in Thin Flake VTe 2. Nano Lett. 20, 4932-4938 (2020).

64. T. E. Karam, J. Hu, G. A. Blake, Strongly Coupled Electron-Phonon Dynamics in FewLayer TiSe 2 Exfoliates. ACS Photonics 5, 1228-1234 (2018).

65. S. Z. Ji, O. Granas, K. Rossnagel, J. Weissenrieder, Transient Three-Dimensional Structural Dynamics in 1T-TaSe2. Phys. Rev. B 101, 094303 (2020).

66. A. J. McKenna, J. K. Eliason, D. J. Flannigan, Spatiotemporal Evolution of Coherent Elastic Strain Waves in a Single $\mathrm{MoS}_{2}$ Flake. Nano Lett. 17, 3952-3958 (2017).

67. A. Singer, S. K. K. Patel, R. Kukreja, V. Uhlíŕ, J. Wingert, S. Festersen, D. Zhu, J. M. Glownia, H. T. Lemke, S. Nelson, M. Kozina, K. Rossnagel, M. Bauer, B. M. Murphy, O. M. Magnussen, E. E. Fullerton, O. G. Shpyrko, Photoinduced Enhancement of the Charge Density Wave Amplitude. Phys. Rev. Lett. 117, 056401 (2016).

68. M. O. Robbins, E. A. Marseglia, X-ray Studies of the Charge-Density Wave Transitions in $\mathrm{TaS}_{2}$. Philos. Mag. B 42, 705-715 (1980). 
69. G. Benedek, G. Brusdeylins, F. Hofmann, P. Ruggerone, J. P. Toennies, R. Vollmer, J. G. Skofronick, Strong Coupling of Rayleigh Phonons to Charge Density Waves in 1T-TaS 2 . Surf. Sci. 304, 185-190 (1994).

70. W. G. Stirling, B. Dorner, J. D. N. Cheeke, J. Revelli, Acoustic Phonons in the TransitionMetal Dichalcogenide Layer Compound, TiSe 2 . Solid State Commun. 18, 931-933 (1976).

71. G. Benedek, L. Miglio, G. Brusdeylins, C. Heimlich, J. G. Skofronick, J. P. Toennies, Surface Phonon Dynamics in 2H-TaSe 2 (001). Europhys. Lett. 5, 253-258 (1988).

72. A. Singer, J. G. Ramirez, I. Valmianski, D. Cela, N. Hua, R. Kukreja, J. Wingert, O. Kovalchuk, J. M. Glownia, M. Sikorski, M. Chollet, M. Holt, I. K. Schuller, O. G. Shpyrko, Nonequilibrium Phase Precursors during a Photoexcited Insulator-to-Metal Transition in $\mathrm{V}_{2} \mathrm{O}_{3}$. Phys. Rev. Lett. 120, 207601 (2018).

73. P. Baum, D.-S. Yang, A. H. Zewail, 4D Visualization of Transitional Structures in Phase Transformations by Electron Diffraction. Science 318, 788-792 (2007).

74. M. S. Skolnick, S. Roth, H. Alms, Ultrasound Measurements in the Layered TransitionMetal Dichalcogenides $\mathrm{NbSe}_{2}$ and $\mathrm{TaS}_{2}$. J. Phys. C: Solid State Phys. 10, 2523-2526 (1977).

75. D. E. Moncton, J. D. Axe, F. J. DiSalvo, Neutron Scattering Study of the Charge-Density Wave Transitions in 2H-TaSe 2 and $2 H-\mathrm{NbSe}_{2}$. Phys. Rev. B 16, 801-819 (1977).

76. K. Ohtake, E. Sato, Y. Suzuki, R. Yamamoto, M. Doyama, K. Endo, S. Wakayama, T. Kishi, Acoustic Emission During CDW Transition in $1 \mathrm{~T}-\mathrm{TaS}_{2}$ and $\left(\mathrm{TaSe}_{4}\right)_{2} \mathrm{I}$. J. Phys. Colloques 46, 681-684 (1985).

77. T.-R. T. Han, F. Zhou, C. D. Malliakas, P. M. Duxbury, S. D. Mahanti, M. G. Kanatzidis, C.-Y. Ruan, Exploration of Metastability and Hidden Phases in Correlated Electron Crystals Visualized By Femtosecond Optical Doping and Electron Crystallography. Sci. Adv. 1, e1400173 (2015).

78. T. Danz, T. Domröse, C. Ropers, Ultrafast Nanoimaging of the Order Parameter in a Structural Phase Transition. Science 371, 371-374 (2021).

79. A. Zong, X. Z. Shen, A. Kogar, L. D. Ye, C. Marks, D. Chowdhury, T. Rohwer, B. Freelon, S. Weathersby, R. K. Li, J. Yang, J. Checkelsky, X. J. Wang, N. Gedik, Ultrafast Manipulation of Mirror Domain Walls in a Charge Density Wave. Sci. Adv. 4, eaau5501 (2018).

80. V. L. R. Jacques, C. Laulhé, N. Moisan, S. Ravy, D. Le Bolloc'h, Laser-Induced ChargeDensity-Wave Transient Depinning in Chromium. Phys. Rev. Lett. 117, 156401 (2016).

81. S. H. Sung, N. Schnitzer, L. Brown, J. Park, R. Hovden, Stacking, Strain, and Twist in 2D Materials Quantified by 3D Electron Diffraction. Phys. Rev. Mater. 3, 064003 (2019). 


\title{
Supporting Information for
}

\section{Coherent Phonon Disruption and Lock-In During a Photoinduced Charge-Density-Wave Phase Transition}

\author{
Spencer A. Reisbick ${ }^{\dagger}$, Yichao Zhang, Jialiang Chen, Paige E. Engen, and David J. Flannigan* \\ Department of Chemical Engineering and Materials Science, University of Minnesota, 421 \\ Washington Ave. SE, Minneapolis, Minnesota 55455, United States \\ *Author to whom correspondence should be addressed. \\ Email: flan0076@umn.edu \\ Office: 612-625-3867 \\ †Present affiliation: Brookhaven National Laboratory, Upton, NY
}

\section{Kinematic determination of real-space TA distortion from $I_{h k}$.}

Out-of-plane lattice dynamics due to the TA component of the hybrid CAP mode was determined from $I_{\overline{1} \overline{1} 0}$ response. Kinematically, $\Delta I_{\overline{1} \overline{1} 0}$ arises from local rocking of the relrods as the phonon wavetrain propagates across the probed region. $I_{\overline{1}} \overline{1} 0$ then describes the Ewald sphere interaction with the reciprocal lattice, as quantified in Equation S1 (81).

$$
I_{h k l}=4 \frac{\sin ^{2}\left(\frac{1}{2} k_{z} z_{0}\right)}{\sin ^{2}\left(\frac{1}{2} k_{z} d_{0}\right)}
$$

Here, $z_{0}$ is the specimen thickness, $d_{\mathrm{o}}$ is the thickness of each $\mathrm{TaS}_{2}$ monolayer, and $k_{z}$ is the parameterized radius of the Ewald sphere. The radius of the Ewald sphere is the magnitude of the incident electron wave vector $\left|\mathbf{k}_{i}\right| \equiv k_{i}$. The Ewald sphere is parameterized with respect to specimen orientation (Equation S2).

$$
k_{z}^{\prime}=k_{i} \cos \theta
$$

Here, $\theta$ is the specimen tilt angle with respect to $\mathbf{k}_{i}$. Here, because slight tilting is present along both the $\alpha$ and $\beta$ axes, an equivalent single-tilt angle is calculated using Equation S3.

$$
\theta=\operatorname{asin}(\sqrt{\sin (\alpha) \cdot \sin (\beta)})
$$

The reciprocal-space coordinates on the $k_{z}=0$ surface are calculated from Equations S4 and S5.

$$
\begin{aligned}
& k_{x}^{\prime}=k_{x}-k_{i} \sin \theta \cos \varphi \\
& k_{y}^{\prime}=k_{y}-k_{i} \sin \theta \sin \varphi
\end{aligned}
$$

Here, $\varphi$ is the in-plane specimen rotation angle. Accordingly, intersection of the Ewald sphere and relrod of interest is described by Equation S6. 


$$
k_{z}=k_{z}^{\prime}-\sqrt{k_{i}-k_{x}^{\prime 2}-k_{y}^{\prime 2}}
$$

Using this approach, the normalized $I_{\overline{1}} \overline{1} 0$ intensity was calculated for tilt angles between $-2^{\circ}$ and $2^{\circ}$ (Fig. S1). The equivalent tilt angle was calculated to be $1.1^{\circ}$, which corresponds to a normalized intensity of 0.292 . The intensities at $\pm 15 \%$ of 0.292 were calculated, and the corresponding tilt angles were determined (Fig. S1 inset). From this, the tilt-angle equivalent of the lattice distortion associated with the $c$-axis polarized TA component is $0.031^{\circ}(0.54 \mathrm{mrad})$.

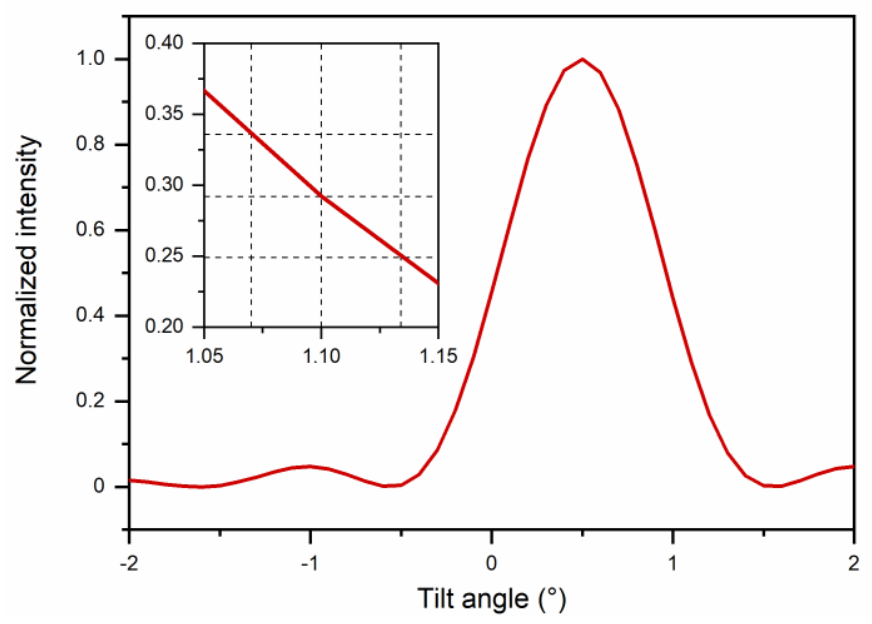

Figure S1. $I_{\overline{1} \overline{1} 0}$ vs. lattice tilt. Normalized $I_{\overline{1} \overline{1} 0}$ as a function of tilt angle for a $66-\mathrm{nm}$ thick $1 T$ $\mathrm{TaS}_{2}$ crystal. Inset is a magnified view of the range spanning $1.05^{\circ}$ to $1.15^{\circ}$. Vertical dashed lines mark the tilt angle prior to photoexcitation and the maximum change in equivalent tilt angle due to TA distortion. Horizontal lines mark the corresponding normalized intensities.

\section{Zone-axis determination.}

Diffraction patterns (DPs) of $1 T-\mathrm{TaS}_{2}$ were simulated spanning all combinations of $\alpha$ and $\beta$ between $0^{\circ}$ and $3^{\circ}$ at $0.1^{\circ}$ steps, where $0^{\circ}$ is exactly on the [001] zone axis (Fig. S2ab). The difference between each simulated DP and an experimental DP was performed, and all results were plotted to generate an $\alpha \beta$ phase map (Fig. S2c). The global minimum (i.e., the smallest difference) occurs at $\alpha=1.5^{\circ}$ and $\beta=0.8^{\circ}$.
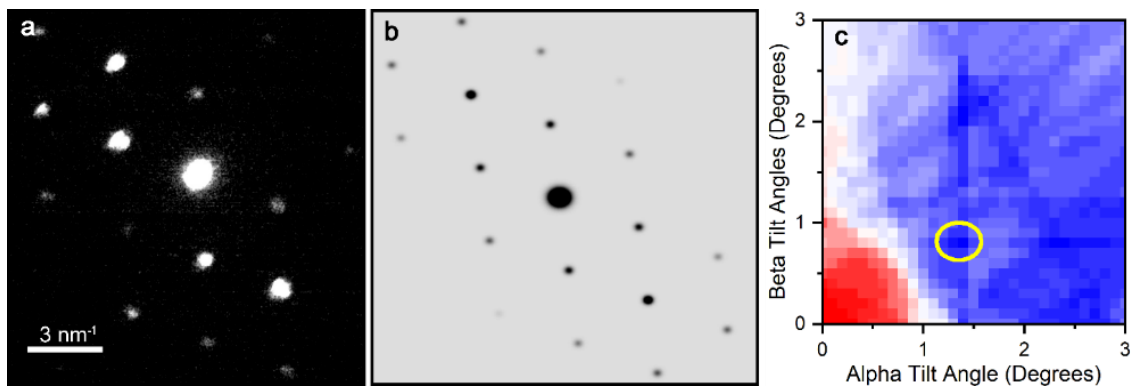

Figure S2. $\mathbf{T a S}_{2}$ specimen orientation relative to $\mathbf{k}_{i}$. (a) Representative experimental DP. (b) Best-matched simulated DP, with $\alpha=1.5^{\circ}$ and $\beta=0.8^{\circ}$ away from the [001] zone axis. (c) $\alpha \beta$ 
phase map generated from 900+ different simulated DPs compared to the experimental DP. Cooler colors $=$ smaller differences. The global minimum is circled. Images generated using CrystalMaker $^{\circledR}$ : a crystal and molecular structures program for Mac and Windows. CrystalMaker Software Ltd, Oxford, England (www.crystalmaker.com).

\section{Photothermal simulations.}

The finite element method embedded in COMSOL Multiphysics ${ }^{\circledR}$ was used to model photothermal heating of the $1 T-\mathrm{TaS}_{2}$ specimen following femtosecond photoexcitation. Laser settings are also reported in the main text. Global specimen geometry for modeling photothermal heating and transport was determined with low-mag TEM imaging, HAADF-STEM imaging, and EELS thickness mapping (47), a schematic of which is shown in Figure S3a. The intensity profile of the pump laser pulse was modeled using Equation S7.

$$
I(x, y, t)=\frac{E_{p u l s e}}{(2 \pi)^{3 / 2} \sigma_{s}^{2} \sigma_{t}} \cdot \exp \left[-\frac{x^{2}+y^{2}}{2 \sigma_{s}^{2}}\right] \cdot \exp \left[-\frac{t^{2}}{2 \sigma_{t}^{2}}\right]
$$

Here, the spatial standard deviation $\sigma_{s}=F W H M_{S} / 2 \sqrt{2 \ln 2}=42.47 \mu \mathrm{m}$, the temporal standard deviation $\sigma_{t}=F W H M_{t} / 2 \sqrt{2 \ln 2}=127.40 f \mathrm{~s}$, and $E_{\text {pulse }}$ denotes the pulse energy.

In the simulation, the laser pulse is treated as a surface heat flux described by Equation S8.

$$
Q(x, y, t, d)=I(x, y, t) \cdot(1-R) \cdot[1-\exp (-\alpha d)]
$$

Here, $d$ is the specimen thickness, $R$ is the reflectivity, and $\alpha$ is the absorption coefficient. The photon energy is $1.2 \mathrm{eV}$, while $R$ and $\alpha$ were 0.5 and $2.5 \times 10^{5} \mathrm{~cm}^{-1}$, respectively, from the measurements by Beal et al. (54).

The time-dependent temperature field is then simulated by solving the heat diffusion equation (Eq. S9).

$$
\rho C_{p} \frac{d T}{d t}=\nabla \cdot(\kappa \nabla T)+Q(x, y, t, d)
$$

Here, the mass density $\rho$ of $1 T-\mathrm{TaS}_{2}$ is $6.86 \mathrm{~g} / \mathrm{cm}^{3}$, the specific heat $C_{p}$ and thermal conductivity $\kappa$ are $420 \mathrm{~J} /(\mathrm{kg} \cdot \mathrm{K})$ and $5 \mathrm{~W} /(\mathrm{m} \cdot \mathrm{K})$, respectively $(55,56)$. The initial specimen temperature is set to $293.15 \mathrm{~K}$. The surface of the specimen is in contact with the $\mathrm{Cu}$ support grid at two of the four sides, and this is assumed to be an open boundary and have a constant temperature initially in equilibrium with the ground-state specimen.

As noted in the main text, a single-temperature model was used owing to the low $\kappa$ of the material resulting in extremely tight tolerances. Thus, attempted use of a two-temperature model led to unphysical divergences. This has little real consequence here owing to our focus on the CAP mode relaxation channel and its correlation to the NC-to-IC phase transition. A temperature contour plot at $t=0$ (set as the peak position of the pump pulse) was generated (Fig. S3b). The initial global temperature rise in the specimen is homogeneous except for enhanced heating at a 26-nm terrace running approximately from the upper-left to the lower-right of the flake. Note that a 
homogeneous response is expected owing to the $100-\mu \mathrm{m}$ pump spot size. The ROI probed is located in the bottom section of the 66-nm thick section.

With no substantial spatial variance in the simulated temperature field, we used an average specimen temperature to represent the temporal evolution. Figure S3c thus shows the average photothermal temperature rise in the first 5 ps following photoexcitation with an absorbed 4$\mathrm{mJ} / \mathrm{cm}^{2}$ pump fluence. The temperature rise begins during the pump pulse and rises to $479.18 \mathrm{~K}$ in $\sim 1 \mathrm{ps}$. The specimen temperature drops to only $478.96 \mathrm{~K}$ during the first $50 \mathrm{ps}$ (not shown) and by only $\sim 2 \mathrm{~K}$ within the first nanosecond.

The full relaxation profile was fit with a single exponential decay (Eq. S10) to determine the recovery time.

$$
T(t)=T_{0}+\Delta T \cdot \exp \left[-\frac{t-t_{0}}{\tau}\right]
$$

Here, $\tau$ is the decay time constant and is $86.3 \mathrm{~ns}$, and $T_{0}=293.15 \mathrm{~K}$. Complete photothermal energy dissipation from the flake and recovery to $T_{0}$ is complete within $800 \mathrm{~ns}$ (Fig. S3d). Thus, for the repetition rate used here $(10 \mathrm{kHz})$, full recovery occurs between each pulse and no average temperature rise is expected to occur.
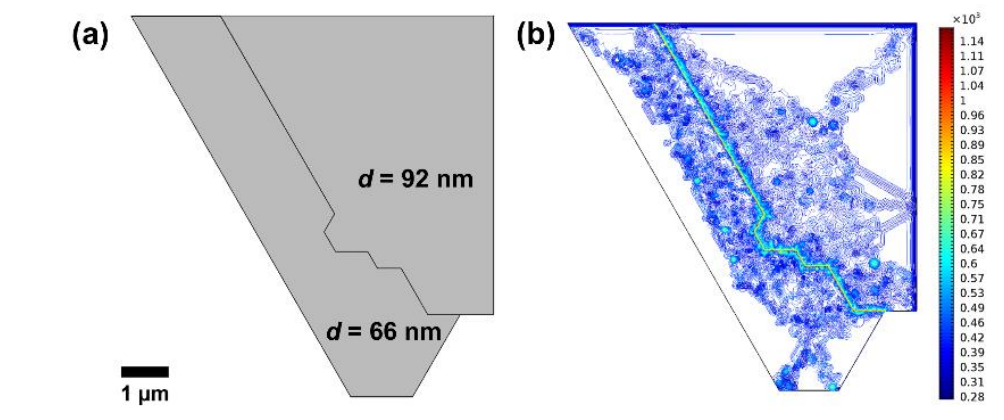

(c)

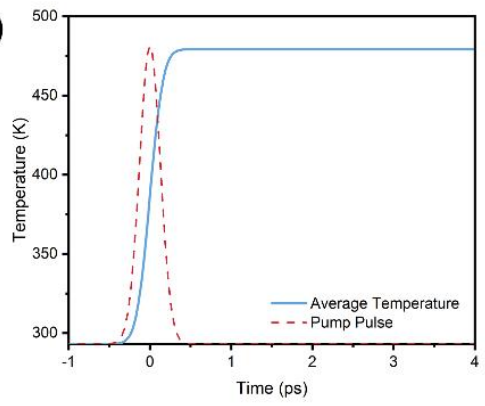

(d)

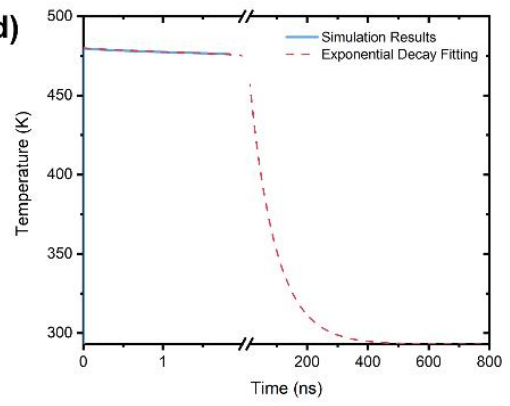

Figure S3. Photothermal modeling of the 1T-TaS 2 flake. (a) Model geometry of the $1 T-\mathrm{TaS}_{2}$ specimen constructed with COMSOL Multiphysics ${ }^{\circledR}$. (b) Corresponding temperature contour plot at $t=0$ ps. (c) Simulated evolution of the average temperature over the specimen in the first $5 \mathrm{ps}$ of the photothermal process (-1 ps to $4 \mathrm{ps}$ ). The red dashed line indicates the normalized temporal profile of the pump pulse. (d) Single-exponential temperature decay out to $t=800 \mathrm{~ns}$. The blue solid line represents the simulation results of the first $2 \mathrm{~ns}$, and the red-dashed curve is an extrapolation of the fitting function. The fitting curve reaches $293.15 \mathrm{~K}$ at $\sim 800 \mathrm{~ns}$. 